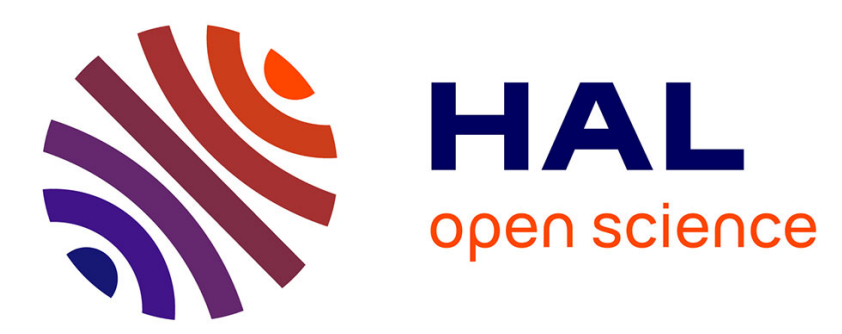

\title{
A shallow water model for the numerical simulation of overland flow on surfaces with ridges and furrows
}

Ulrich Razafison, Stephane Cordier, Olivier Delestre, Frédéric Darboux, Carine Lucas, Francois James

\section{- To cite this version:}

Ulrich Razafison, Stephane Cordier, Olivier Delestre, Frédéric Darboux, Carine Lucas, et al.. A shallow water model for the numerical simulation of overland flow on surfaces with ridges and furrows. European Journal of Mechanics - B/Fluids, 2012, 31 (1), pp.44-52. 10.1016/j.euromechflu.2011.07.002 . hal-00429152v2

\section{HAL Id: hal-00429152 \\ https://hal.science/hal-00429152v2}

Submitted on 8 Feb 2011

HAL is a multi-disciplinary open access archive for the deposit and dissemination of scientific research documents, whether they are published or not. The documents may come from teaching and research institutions in France or abroad, or from public or private research centers.
L'archive ouverte pluridisciplinaire HAL, est destinée au dépôt et à la diffusion de documents scientifiques de niveau recherche, publiés ou non, émanant des établissements d'enseignement et de recherche français ou étrangers, des laboratoires publics ou privés. 


\title{
A shallow water model for the numerical simulation of overland flow on surfaces with ridges and furrows
}

\author{
Ulrich Razafison $^{1}$, Stéphane Cordier ${ }^{2}$, Olivier Delestre ${ }^{2}$, Frédéric Darboux ${ }^{3}$, \\ Carine Lucas ${ }^{2}$ and François James ${ }^{2}$ \\ 1 - Université de Franche-Comté, Laboratoire de Mathématiques, CNRS UMR \\ 6623, 16 route de Gray, 25030 Besançon Cedex, France \\ 2 - Université d'Orléans, Laboratoire MAPMO, CNRS UMR 6628, Fédération \\ Denis-Poisson, FR CNRS 2964, B. P. 6759, 45067 Orléans Cedex 2, France \\ 3 - INRA, UR 0272 Science du sol, Centre de recherche d'Orléans, CS 40001, \\ F-45075 Orléans Cedex 2, France \\ Email adresses: ulrich.razafison@math.cnrs.fr, stephane.cordier@univ-orleans.fr, \\ olivierdelestre41@yahoo.fr, frederic.darboux@orleans.inra.fr, \\ francois.james@univ-orleans.fr, carine.lucas@univ-orleans.fr,
}

\begin{abstract}
Keywords: Overland flow, Shallow water equations, Furrows, Friction We introduce here a new Shallow Water model for the numerical simulation of overland flow with furrows effects without representing them explicitly. The model is obtained by adding to the classical Shallow Water equations an anisotropic friction term that takes into account these effects.

We validate the model with numerical tests and we compare it with the classical Shallow Water model where the furrows are explicitly and precisely described.
\end{abstract}

AMS Classification: 93A30, 81T80, 58J45, 35L65

\section{Introduction}

During rainfalls, overland flow on cultivated lands induces problems at watershed scale for soil conservation (decrease of soil thickness by erosion, nutrient losses), infrastructures (flooding and destruction of roads and buildings), preservation of water quality (drinking water) and sustainability of aquatic ecosystems (chemical pollution).

These troubles can be prevented by improving watershed management in connection with overland flow. This requires to simulate well the water flux at the outlet but also to have 
a good prediction of the spatial distribution of the water flux and velocity over the whole watershed. However, the current hydrological models have a low efficiency in predicting overland flow within small watersheds (see $[4,20,21]$ ). In agricultural watersheds, one of the main difficulties is that flow directions are controlled not only by the topography, but also, by ditches along the field boundaries, and, inside the fields, by ridges and furrows created by tillage operations. The flow pattern is clearly the result of the interaction between these objects [28], but the way they interact remains mostly unspecified. Therefore, one must improve the understanding of this interaction in order to better predict the spatial and temporal distribution of overland flow and so to improve the decisions made by watershed managers.

In this paper we focus on the interaction between topography and furrows, a feature encountered in almost all cultivated lands. This interaction can be seen as the interaction between three types of roughness. The topography is the roughness of the Earth and is described in Digital Elevation Maps with a horizontal resolution larger than one meter, and commonly of ten meters and more. The furrows are the roughness due to agricultural practices and create a strong directional heterogeneity inside a field. They are characterized by their wavelength (of about one to a few decimeters), their amplitude (of a few centimeters to one decimeter) and their direction. Finally the random roughness, due to soil aggregates and clods, is homogeneous in space and has an amplitude of a few millimeters to about one decimeter. To our knowledge, most of the works on the interaction between roughness and flow have been dedicated to topography (see [26, 30]) or to random roughness (see $[9,22,18]$ ).

Few works are dealing with furrows, and among them, most are concerned with the storage capacity of the furrows, i.e. the amount of water stored in the puddles created by the furrows (for instance [25]). These works do not consider the water flowing on the soil surfaces but only the water stored in puddles. The few works considering both overland flow and the furrows-topography interaction are empirical studies [28, 29]. They lead to empirical laws giving an on/off prediction: the predicted flow direction is either the direction of the topographic slope or the furrow direction, while in reality water can flow in both directions at the same time. Moreover, these laws are limited by their empirical basis.

To be of practical use, a model accounting for the effects of furrows on overland flow direction must not require an explicit representation of the furrows: that would imply the use of a digital topographic map with a horizontal resolution of about a centimeter for the whole watershed, which already covers an area of about one square kilometer for a small one. Such digital maps are not available and, even if available, will require too much computation resources. 
The purpose of this work is to propose a model that is able to take into account the effects of the furrows on overland flow. Numerical results are presented. The model is a first step in an attempt to predict overland flow directions controlled by furrows and topography without representing the furrows explicitly. Indeed, the furrows are known only trough their average amplitude, wavelength and direction. In this paper, the furrow direction is kept perpendicular to the slope. Our model is based on the Shallow Water equations that are widely used to describe flows in rivers, in ocean and overland flow among other applications.

The outline of the paper is as follows. In the next section, we present first the Shallow Water model. Then we propose a new model where we add a new friction term to account for the effects of the furrows on overland flow. Section 3 describes the numerical scheme used to solve the model, and, in section 4, we present and discuss the numerical results that we obtain with our model. Conclusions are outlined in Section 5.

\section{The mathematical models}

The starting point is the 2D classic Shallow Water system [10] in a bounded domain $\Omega$ :

$$
\left\{\begin{array}{l}
\frac{\partial h}{\partial t}+\frac{\partial(h u)}{\partial x}+\frac{\partial(h v)}{\partial y}=R \\
\frac{\partial(h u)}{\partial t}+\frac{\partial\left(h u^{2}\right)}{\partial x}+\frac{\partial(h u v)}{\partial y}+g h \frac{\partial h}{\partial x}+g h \frac{\partial Z}{\partial x}+g k^{2} h^{-1 / 3}|\boldsymbol{u}| u=0 \\
\frac{\partial(h v)}{\partial t}+\frac{\partial(h u v)}{\partial x}+\frac{\partial\left(h v^{2}\right)}{\partial y}+g h \frac{\partial h}{\partial y}+g h \frac{\partial Z}{\partial y}+g k^{2} h^{-1 / 3}|\boldsymbol{u}| v=0
\end{array}\right.
$$

For $t>0$ and $\boldsymbol{x}=(x, y) \in \Omega$, the unknowns are the water height $h=h(t, \boldsymbol{x})$ and the horizontal flow velocity $\boldsymbol{u}=\boldsymbol{u}(t, \boldsymbol{x})=(u(t, \boldsymbol{x}), v(t, \boldsymbol{x}))^{T}$. Furthermore, $Z(\boldsymbol{x})$ describes the bottom topography of the domain and therefore $h+Z$ is the level of the water surface (see Figure 1). In equations (2.1), $g$ is the acceleration due to the gravity and $R$ is the rainfall intensity. We refer for instance to $[17,15,24]$ for a derivation of the Shallow Water system originating from the free surface Navier-Stokes equations.

For the friction term, we choose the Manning law with $k$ the Manning coefficient. We also denote $\boldsymbol{q}(t, \boldsymbol{x})=\left(q_{x}(t, \boldsymbol{x}), q_{y}(t, \boldsymbol{x})\right)^{T}=h(t, \boldsymbol{x}) \boldsymbol{u}(t, \boldsymbol{x})$ the water flux.

Now we consider a rectangular domain $\Omega=\ell \times L$ and a topography $Z$ with furrows. We suppose that the topography is an inclined plane with sinus furrows, and that the geometry of the furrows is known through their amplitude and their wavelength. Note that realistic (measured) furrows will be a slightly different shape due the existence of random roughness. However, random roughness, being isotropic, does not affect flow direction at the scale of the furrows. We also suppose that the furrows are perpendicular 


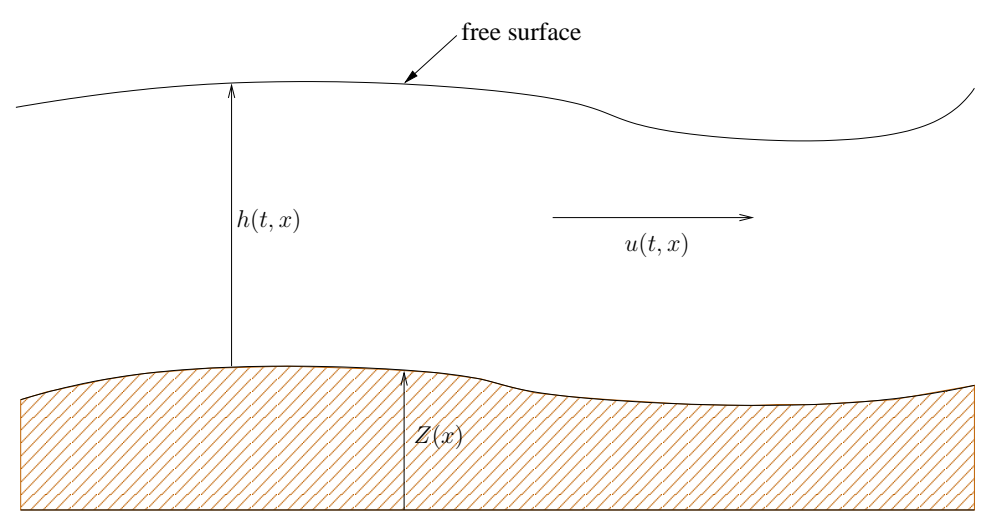

Figure 1: Notations for a 1D Shallow Water flow

to the length of $\Omega$ with respect to $y$. An example of such a topography is illustrated in Figure 2.

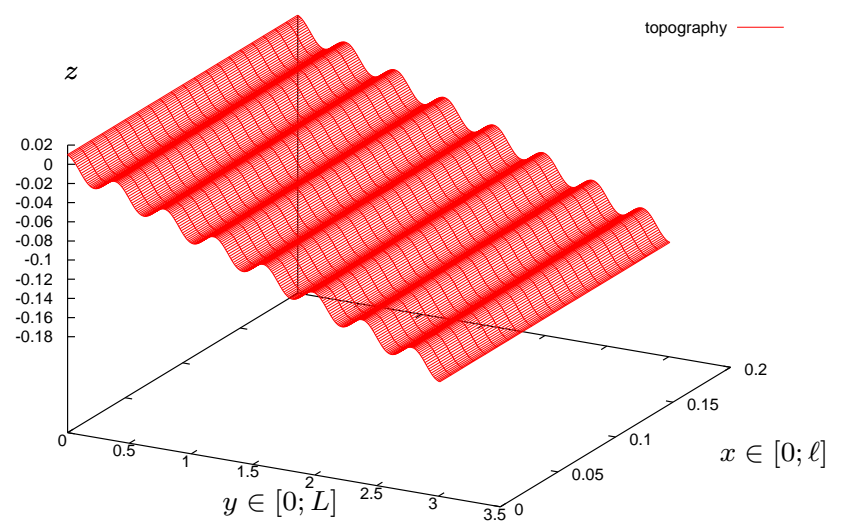

Figure 2: An example of a topography with furrows that we consider

Next, we shall complement the problem with the following assumptions.

1. The direction of the flow is parallel to the length of the domain $\Omega$ with respect to $y$ (pseudo-1D case) and consequently perpendicular to the furrows.

2. We only consider fluvial flows which means that $|\boldsymbol{u}|<\sqrt{g h}$.

3. Infiltration and soil erosion are not taken into account.

Under such assumptions, the furrows overflow at the same time during rainfall events or one after the other in the case of an inflow from upstream.

We aim at proposing a model that takes into account the effects of the furrows without explicitly representing them in the topography $Z$. In other words, we want to find an 
equivalent model to the Shallow Water system on $\Omega$, that would be used at a macroscopic scale, i.e. on a topography which is only an inclined plane. We want to force the flow to slow down when its depth is smaller than the value corresponding to the water height that can be trapped in the furrows. The idea of this article is to model this effect caused by the furrows through an additional friction term that forces the flow to slow down for small water depth. To that end, we first introduce $\left\langle h_{F}\right\rangle$ the average height of the water trapped in the furrows. This value is given by

$$
\left\langle h_{F}\right\rangle=V /\left(L_{F} \times \ell\right) \quad[\mathrm{m}]
$$

where $V$ is the volume of trapped water in a furrow, $L_{F}$ its wavelength (see Figure 3 ) and $\ell$ is the length of the domain $\Omega$ (with respect to $x$ ). Note that the value of $\left\langle h_{F}\right\rangle$ only depends on three parameters: the slope of the domain, the furrows average amplitude and the furrows average wavelength.

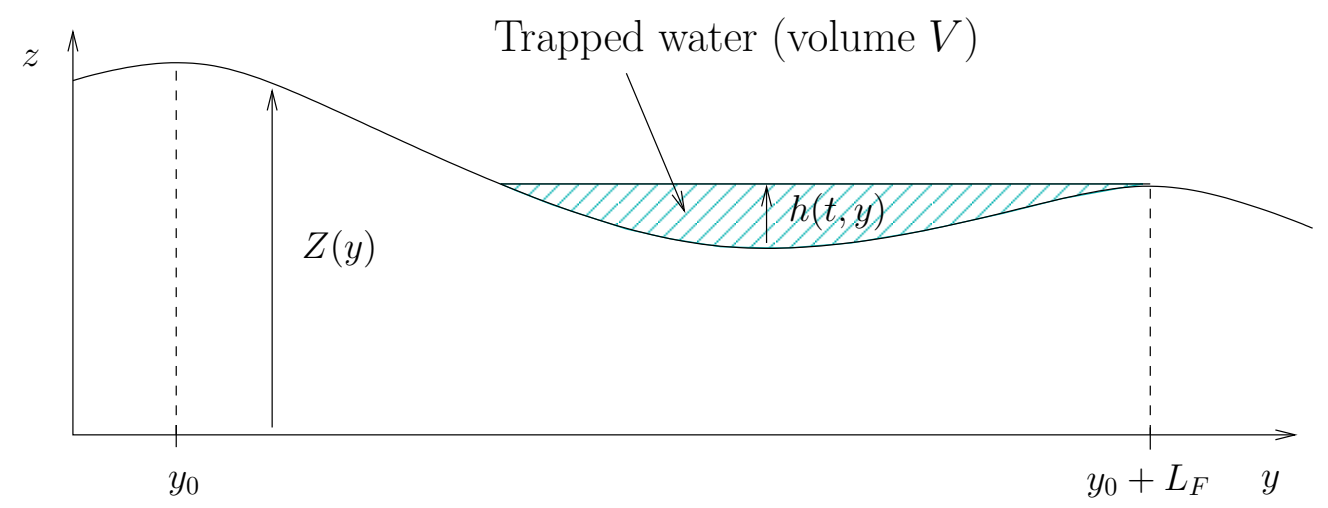

Figure 3: Water trapped in a furrow

Next we consider the following additional friction coefficient:

$$
K(h)=K_{0} \exp \left(\frac{-h+\left\langle h_{F}\right\rangle}{C\left\langle h_{F}\right\rangle}\right)
$$

where $C$ is a characteristic constant, increasing function of the small random variations of the height of the furrows and $K_{0}$ is a coefficient we determine in the following.

In Figure 4, the general shape of $K(h)$ is plotted for $\left\langle h_{F}\right\rangle=0.01 \mathrm{~m}$. We clearly see that $K(h)$ is large for $h \leq\left\langle h_{F}\right\rangle$. This shows that when the water height $h$ is lower than the average height of the furrows $\left\langle h_{F}\right\rangle$ then, thanks to $K(h)$, the flow is slowed down.

Remark 2.1. Let us explain in a few words relation (2.3).

1. If $\left\langle h_{F}\right\rangle$ tends to 0 , then $K(h)$ also tends to 0 for any $h>0$. In other words, the additional friction coefficient disappears when there are no furrows. 


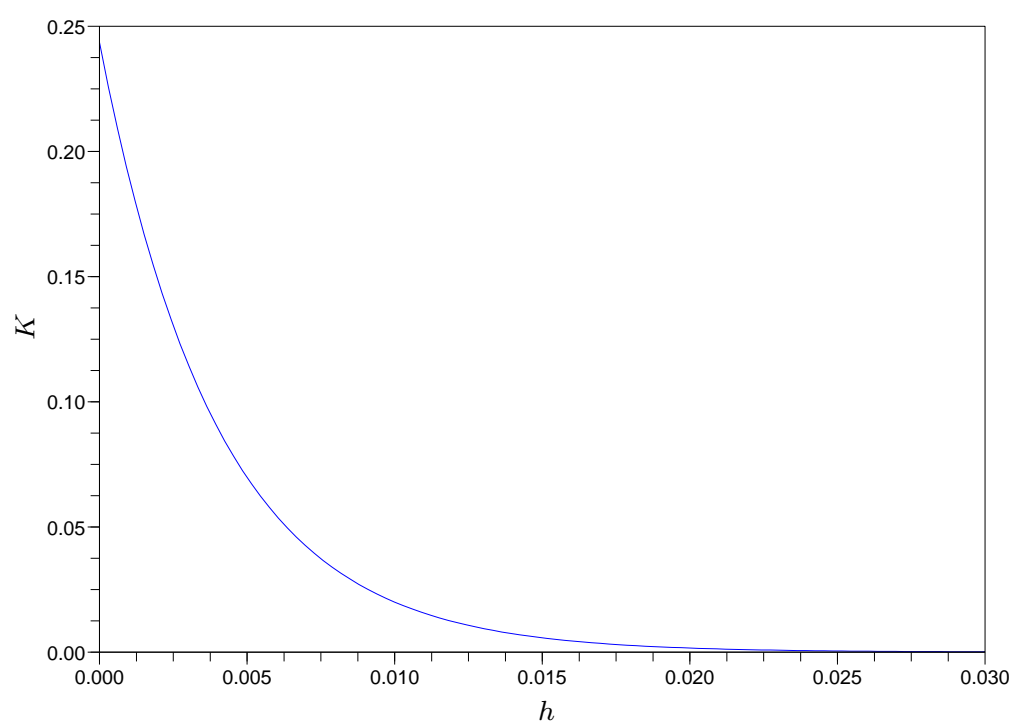

Figure 4: Shape of the friction term $K(h)$ for $\left\langle h_{F}\right\rangle=0.01 \mathrm{~m}$

2. If $C$ tends to 0 , then we obtain the empirical models that are usually used. These models consist in giving an on/off prediction of the furrows-topography interaction (see $[28,29]$ ); more precisely, while the critical water height is not attained, there is no flow, and after this threshold, the furrows are not taken into account.

The new Shallow Water model we introduce here (see (2.4)) can be seen as an improvement of these models.

Finally, we propose the following new Shallow Water model with a "furrows-friction" coefficient:

$$
\left\{\begin{array}{l}
\frac{\partial h}{\partial t}+\frac{\partial(h u)}{\partial x}+\frac{\partial(h v)}{\partial y}=R \\
\frac{\partial(h u)}{\partial t}+\frac{\partial\left(h u^{2}\right)}{\partial x}+\frac{\partial(h u v)}{\partial y}+g h \frac{\partial h}{\partial x}+g h \frac{\partial Z}{\partial x}+g k^{2} h^{-1 / 3}|\boldsymbol{u}| u=0 \\
\frac{\partial(h v)}{\partial t}+\frac{\partial(h u v)}{\partial x}+\frac{\partial\left(h v^{2}\right)}{\partial y}+g h \frac{\partial h}{\partial y}+g h \frac{\partial Z}{\partial y}+g k^{2} h^{-1 / 3}|\boldsymbol{u}| v+K(h) h v=0
\end{array}\right.
$$

Remark 2.2. 1. Note that, since the furrows are perpendicular to the slope, the additional friction law $K(h) h v$ only appears in the third equation of (2.4) and therefore, it only acts on the flow in the $y$-axis direction. This assumption is not restrictive: in general, the direction of the furrows is constant on each agricultural field, and, if necessary, we apply a rotation to get the equations for an arbitrary direction of the furrows. 
2. The form of the new friction law is arbitrarily chosen. The general form of friction laws is $K h^{\alpha}|\boldsymbol{u}|^{\beta} \boldsymbol{u}$ where $\alpha$ and $\beta$ are positive real numbers. For example we get Manning's law for $(\alpha, \beta)=(-1 / 3,1)$ and Darcy-Weisbach's law for $(\alpha, \beta)=(1,1)$. Note that these laws are empirical, are obtained considering stationary flows and that their validity is still discussed among hydrologists (e.g. [27]).

For the numerical experiments presented in section 4 with system (2.4), we chose $(\alpha, \beta)=(1,0)$ but, we could have made another choice, should we change the value of $K_{0}$ or the form of $K(h)$.

At this point, let us mention that, since shallow water flows can also be described by the so-called multi-layer Shallow Water system (see for instance to $[1,3,8]$ for a derivation and numerical studies), we can propose another approach based on multi-layer models to take into account the effects of the furrows on overland flows.

In this work, we introduce the following two-layer like model:

$$
\left\{\begin{array}{l}
\text { if } h(t, \boldsymbol{x}) \leq\left\langle h_{F}\right\rangle, \text { then } \boldsymbol{u}(t, \boldsymbol{x})=\mathbf{0} \text { and } h(t, \boldsymbol{x})=R t \\
\text { if } h(t, \boldsymbol{x})>\left\langle h_{F}\right\rangle, \text { then solve }(2.1) \text { with an inclined plane topography. }
\end{array}\right.
$$

In (2.5), the lower layer corresponds to the filling up of the furrows; note that the upper layer is active only when the furrows overflow. The initial conditions for the upper layer are then $\boldsymbol{u}(0, \boldsymbol{x})=\mathbf{0}$ and $h(0, \boldsymbol{x})=\hat{h}-\left\langle h_{F}\right\rangle$, where $\hat{h}$ is the water height at the overflow time. Note that, in one dimension, this model provides more satisfactory results than the model (2.4) (see section 4). But its extension to more complex two-dimensional problems requires a careful modelling of the coupling between the two layers and it is more difficult than the extension of the model (2.4).

\section{Numerical results}

In this section, we explain the numerical scheme we used in our numerical simulations. The Shallow Water system is not so easy to solve. In hydrology, Mac Cormack scheme is usually used for overland flow simulation (see among others $[14,16]$ ). But it is not well adapted to this system because of several problems, such as, for example, the preservation of the positivity of the water height and of the steady states or the behavior at the wet/dry interface. To that end, we used well-balanced schemes (so-called since [19]) based on the hydrostatic reconstruction $[2,5]$. This finite volume scheme has shown to be adapted to overland flow simulation at small scales $[12,13,11]$.

To make this presentation simpler, we describe the numerical scheme on the classical the one-dimensional Shallow Water model with variable topography and Manning's friction 
law :

$$
\left\{\begin{array}{l}
\frac{\partial h}{\partial t}+\frac{\partial(h u)}{\partial x}=R \\
\frac{\partial(h u)}{\partial t}+\frac{\partial}{\partial x}\left(h u^{2}+g \frac{h^{2}}{2}\right)=-g h \frac{\partial Z}{\partial x}-g k^{2} h^{-1 / 3}|u| u
\end{array}\right.
$$

The model (3.6) can be written into a conservative form

$$
\frac{\partial U}{\partial t}+\frac{\partial F(U)}{\partial x}=S_{0}(U)+S_{f}(U)
$$

where

$$
\begin{gathered}
U=\left(\begin{array}{c}
h \\
h u
\end{array}\right)=\left(\begin{array}{l}
h \\
q
\end{array}\right), \quad F(U)=\left(\begin{array}{c}
h u \\
h u^{2}+g \frac{h^{2}}{2}
\end{array}\right), \\
S_{0}(U)=\left(\begin{array}{c}
R \\
-g h \frac{\partial Z}{\partial x}
\end{array}\right) \quad \text { and } \quad S_{f}(U)=\left(\begin{array}{c}
0 \\
-g k^{2} h^{-1 / 3}|u| u
\end{array}\right) .
\end{gathered}
$$

System (3.7) is discretized using finite volume method for hyperbolic conservation laws. We introduce a space-time grid where the space and the time steps are respectively $\Delta x$ and $\Delta t$. We set $x_{i}=i \Delta x, t^{n}=n \Delta t$ and $\left.C_{i}=\right] x_{i-1 / 2}, x_{i+1 / 2}\left[\right.$. We denote by $U_{i}^{n}$ the approximation of the average of $U\left(t^{n}, x\right)$ over the cell $C_{i}$, namely,

$$
U_{i}^{n} \simeq \frac{1}{\Delta x} \int_{C_{i}} U\left(t^{n}, x\right) d x
$$

Considering for the moment only the homogeneous part of (3.7), then the finite volume scheme is of the form

$$
U_{i}^{n+1}-U_{i}^{n}+\frac{\Delta t}{\Delta x}\left(F_{i+1 / 2}^{n}-F_{i-1 / 2}^{n}\right)=0,
$$

where $F_{i+1 / 2}^{n}=\mathcal{F}\left(U_{i}^{n}, U_{i+1}^{n}\right)$ is the HLL numerical flux (see for instance [5]) through the interface between $C_{i}$ and $C_{i+1}$. Note that the HLL flux is defined by

$$
\mathcal{F}\left(U_{l}, U_{r}\right)= \begin{cases}F\left(U_{l}\right) & \text { if } 0<c_{1}, \\ \frac{c_{1} F\left(U_{l}\right)-c_{2} F\left(U_{r}\right)}{c_{2}-c_{1}}+\frac{c_{1} c_{2}}{c_{2}-c_{1}}\left(U_{r}-U_{l}\right) & \text { if } c_{1}<0<c_{2}, \\ F\left(U_{r}\right) & \text { if } c_{2}<0,\end{cases}
$$

where $c_{1}<c_{2}$ are given by $c_{1}=\inf _{U=U_{l}, U_{r}}\left(\inf _{j=1,2} \lambda_{j}(U)\right), c_{2}=\sup _{U=U_{l}, U_{r}}\left(\sup _{j=1,2} \lambda_{j}(U)\right)$ and where $\lambda_{1}(U)=u-\sqrt{g h}, \lambda_{2}(U)=u+\sqrt{g h}$ are the eigenvalues of the Jabobian matrix $F^{\prime}(U)$.

In order to have a second order accuracy scheme, we use the modified ENO reconstruction (see [6]) defined as follows

$$
\begin{array}{ll}
h_{i-1 / 2+}=h_{i}-\frac{\Delta x}{2} D_{\text {enom }} h_{i}, & h_{i+1 / 2-}=h_{i}+\frac{\Delta x}{2} D_{\text {enom }} h_{i}, \\
u_{i-1 / 2+}=u_{i}-\frac{h_{i+1 / 2-}}{h_{i}} \frac{\Delta x}{2} D_{\text {enom }} u_{i}, & u_{i+1 / 2-}=u_{i}+\frac{h_{i-1 / 2+}}{h_{i}} \frac{\Delta x}{2} D_{\text {enom }} u_{i}
\end{array}
$$


with, for a spatially discretized function $s$,

$$
D_{\text {enom }} s_{i}=\operatorname{minmod}\left(D_{\text {eno }} s_{i}, 2 \theta_{\text {enom }} D_{m m} s_{i}\right)
$$

where

$$
\begin{gathered}
\operatorname{minmod}(x, y)= \begin{cases}\min (x, y) & \text { if } x, y \geqslant 0 \\
\max (x, y) & \text { if } x, y \leqslant 0 \\
0 & \text { otherwise, }\end{cases} \\
D_{\text {eno }} s_{i}=\operatorname{minmod}\left(\frac{s_{i}-s_{i-1}}{\Delta x}+\theta_{\text {eno }} \frac{\Delta x}{2} D^{2} s_{i-1 / 2}, \frac{s_{i+1}-s_{i}}{\Delta x}-\theta_{\text {eno }} \frac{\Delta x}{2} D^{2} s_{i+1 / 2}\right), \\
D^{2} s_{i+1 / 2}=\operatorname{minmod}\left(\frac{s_{i+1}-2 s_{i}+s_{i-1}}{\Delta x^{2}}, \frac{s_{i+2}-2 s_{i+1}+s_{i}}{\Delta x^{2}}\right), \\
D_{m m} s_{i}=\operatorname{minmod}\left(\frac{s_{i}-s_{i-1}}{\Delta x}, \frac{s_{i+1}-s_{i}}{\Delta x}\right)
\end{gathered}
$$

with $\theta_{\text {eno }}, \theta_{\text {enom }} \in[0,1]$. Note that for $\theta_{\text {eno }}=0$, this reconstruction is exactly the usual MUSCL reconstruction.

In order to take into account the topography while preserving the steady state of a lake at rest, that is

$$
h+Z=c s t \text { and } u=0,
$$

we use the hydrostatic reconstruction developed in $[2,5]$. First we need to define the reconstructed values $z_{i+1 / 2-}$ and $z_{i-1 / 2+}$ that can be deduced from the reconstructed values $h_{i-1 / 2+}, h_{i-1 / 2+}$ and the following reconstruction of $z+h$ :

$$
(z+h)_{i-1 / 2+}=z_{i}+h_{i}-\frac{\Delta x}{2} D_{\text {enom }}\left(z_{i}+h_{i}\right)
$$

and

$$
(z+h)_{i+1 / 2-}=z_{i}+h_{i}+\frac{\Delta x}{2} D_{\text {enom }}\left(z_{i}+h_{i}\right)
$$

Next the hydrostatic reconstruction consists in defining the following new values:

$$
\begin{aligned}
& h_{i+1 / 2 l}=\max \left(0, h_{i+1 / 2-}+z_{i+1 / 2-}-\max \left(z_{i+1 / 2-}, z_{i+1 / 2+}\right)\right), \\
& h_{i+1 / 2 r}=\max \left(0, h_{i+1 / 2+}+z_{i+1 / 2+}-\max \left(z_{i+1 / 2-}, z_{i+1 / 2+}\right)\right), \\
& U_{i+1 / 2 l}=\left(\begin{array}{c}
h_{i+1 / 2 l} \\
h_{i+1 / 2 l} u_{i+1 / 2-}
\end{array}\right), U_{i+1 / 2 r}=\left(\begin{array}{c}
h_{i+1 / 2 r} \\
h_{i+1 / 2 r} u_{i+1 / 2+}
\end{array}\right) .
\end{aligned}
$$

The positive parts in the definitions of $h_{i+1 / 2 l}$ and $h_{i+1 / 2 r}$ (we have $\left.\max (0, \cdot)\right)$ insure the positivity of the water height. Therefore the scheme can be written into the form

$$
U_{i}^{n+1}-U_{i}^{n}+\frac{\Delta t}{\Delta x}\left(F_{i+1 / 2 l}^{n}-F_{i-1 / 2 r}^{n}-F c_{i}^{n}\right)=0,
$$


where

$$
\begin{aligned}
& F_{i-1 / 2 r}^{n}=\mathcal{F}\left(U_{i-1 / 2 l}^{n}, U_{i-1 / 2 r}^{n}\right)+\left(\begin{array}{c}
0 \\
\frac{g}{2}\left(\left(h_{i-1 / 2+}^{n}\right)^{2}-\left(h_{i-1 / 2 r}^{n}\right)^{2}\right)
\end{array}\right), \\
& F_{i+1 / 2 l}^{n}=\mathcal{F}\left(U_{i+1 / 2 l}^{n}, U_{i+1 / 2 r}^{n}\right)+\left(\begin{array}{c}
0 \\
\frac{g}{2}\left(\left(h_{i+1 / 2-}^{n}\right)^{2}-\left(h_{i+1 / 2 l}^{n}\right)^{2}\right)
\end{array}\right),
\end{aligned}
$$

and

$$
F c_{i}^{n}=\left(\begin{array}{c}
0 \\
-\frac{g}{2}\left(h_{i-1 / 2+}^{n}+h_{i+1 / 2-}^{n}\right)\left(z_{i+1 / 2-}^{n}-z_{i-1 / 2+}^{n}\right)
\end{array}\right) .
$$

The term $F c_{i}^{n}$ is added to obtain a well-balanced and consistent scheme (see [2]). Now in order to have a second order scheme in time, we use the Heun method,

$$
\begin{aligned}
\widetilde{U}_{i}^{n+1} & =U_{i}^{n}+\Delta t \Phi\left(U_{i}^{n}\right) \\
\widetilde{U}_{i}^{n+2} & =\widetilde{U}_{i}^{n+1}+\Delta t \Phi\left(\widetilde{U}_{i}^{n+1}\right) \\
U_{i}^{n+1} & =\frac{U_{i}^{n}+\widetilde{U}_{i}^{n+2}}{2}
\end{aligned}
$$

where

$$
\Phi\left(U_{i}^{n}\right)=\frac{1}{\Delta x}\left(F_{i+1 / 2 l}^{n}-F_{i-1 / 2 r}^{n}-F c_{i}^{n}\right) .
$$

Concerning the Manning friction term, we follow $[16,7]$ and introduce a semi-implicit treatment of this term. Then the scheme is modified as follows

- Solve the Shallow Water system

$$
U_{i}^{*}=U_{i}^{n}+\Delta t \Phi\left(U_{i}^{n}\right)
$$

- Compute $\widetilde{U}_{i}^{n+1}=\left(\begin{array}{c}h_{i}^{n+1} \\ h_{i}^{n+1} \\ \widetilde{u}_{i}^{n+1}\end{array}\right)$ by solving the Manning friction term

$$
\left(\begin{array}{c}
h_{i}^{n+1} \\
h_{i}^{*} \frac{\widetilde{u}_{i}^{n+1}-u_{i}^{*}}{\Delta t}
\end{array}\right)=S f_{\Delta t}\left(U_{i}^{*}\right) \equiv\left(\begin{array}{c}
h_{i}^{*} \\
-g k^{2} \frac{\widetilde{q}_{i}^{n+1}\left|q_{i}^{*}\right|}{h_{i}^{n}\left(h_{i}^{n+1}\right)^{4 / 3}}
\end{array}\right) .
$$

- Solve the Shallow Water system

$$
U_{i}^{* *}=\widetilde{U}_{i}^{n+1}+\Delta t \Phi\left(\widetilde{U}_{i}^{n+1}\right)
$$

- Compute $\widetilde{U}_{i}^{n+2}=\left(\begin{array}{c}h_{i}^{n+2} \\ h_{i}^{n+1} \widetilde{u}_{i}^{n+2}\end{array}\right)$ by solving the Manning friction term

$$
\left(\begin{array}{c}
h_{i}^{n+2} \\
h_{i}^{* *} \frac{\widetilde{u}_{i}^{n+2}-u_{i}^{* *}}{\Delta t}
\end{array}\right)=S f_{\Delta t}\left(U_{i}^{* *}\right) .
$$


- Compute $U_{i}^{n+2}$ using the Heun method defined by (3.8).

We now describe the treatment of the boundary conditions at the inflow and the outflow. We denote by a $b$ subscript the values on the (fictive) boundary cell, and by the index "in" the values in the first cell, inside the domain. The normal $n$ is equal to -1 on the left boundary $(x=0)$ and 1 on the right boundary $(x=\ell)$.

- A solid wall is modeled imposing $u_{b}=-u_{\text {in }}, h_{b}=h_{\text {in }}$, on the condition that the topography be extended horizontally on the fictive cells.

- At the inflow boundary, we impose the discharge $q_{b}$ satisfying $n q_{b}<0$. Since we only consider fluvial flows, the water height $h_{b}$ is computed using Riemann invariants (see for instance $[7,23]$ ). More precisely, assume that $c=\sqrt{g h}$. It is well known that for the Shallow Water system (3.6), the quantity $u \mp 2 c$ is constant along the characteristic $\frac{d x}{d t}=u \mp c$. Thus we have

$$
u_{b}+n \times 2 c_{b}=u_{\text {in }}+n \times 2 c_{\mathrm{in}} .
$$

Multiplying (3.9) by $h_{b}$, we obtain

$$
-n \times 2 \sqrt{g} h_{b}^{3 / 2}+\left(u_{\text {in }}+n \times 2 c_{\text {in }}\right) h_{b}-q_{b}=0 .
$$

Newton method is used to solve this last equation and to get $h_{b}$.

- At the outflow boundary, we always impose the water height $h_{b}$ and, here again, we use Riemann invariants to compute the discharge. We get

$$
u_{b}=u_{\text {in }}+n \times 2\left(c_{\text {in }}-c_{b}\right)
$$

and we can easily deduce $q_{b}=h_{b} u_{b}$.

All these previous steps are quite usual for the resolution of the Shallow Water system (2.1). Note that, for our new $2 \mathrm{D}$ model (2.4), the additional friction term is treated in an explicit way.

\section{Numerical results}

In section 2, we introduced several models: the usual Shallow Water system (2.1), the Shallow Water system with an additional friction coefficient that represents the furrows when we consider a plane topography (2.4) and a two-layer like model (2.5).

In this section, we present several results obtained with these three models in order to show the capacity of our new model (2.4) to approximate the exact solution. Namely, we 
consider two types of test cases: in the first one, we only take into account rainfalls and in the second one the water comes from upstream. For these two numerical experiments, the "exact" (or reference) solution is the one of the Shallow Water system (2.1) with a precise description of a topography with furrows. The domain $\Omega$ we consider here is $\Omega=\ell \times L$, where $\ell=0.2 \mathrm{~m}$ and $L=4 \mathrm{~m}$ (see Figure 2). We assume that the plane topography has a constant slope of $5 \%$. The amplitude of the furrows is $0.01 \mathrm{~m}(0.02 \mathrm{~m}$ peak-to-peak $)$ and their wavelength is $0.1 \mathrm{~m}$. We choose a friction coefficient $k=0.04 \mathrm{~m}^{1 / 3} \mathrm{~s}^{-1}$. For the following computations, we use a time step $\Delta t=0.001 \mathrm{~s}$ (this time step is imposed by the resolution of (2.1), as we need a small space step to get a good representation of the furrows).

Let us mention that all the numerical results are obtained using a $\mathrm{C}++$ software for the resolution of the Shallow Water system, and a new library for the new friction coefficient.

\subsection{Reference solutions and description of the test cases}

This paragraph is devoted to the computation of the solutions of the Shallow Water model (2.1) where the geometry of the furrows is known explicitly. These solutions will be considered here as reference solutions. According to the parameters given above, the topography is modeled by the equation:

$$
Z(x, y)=-0.05 y+0.01 \cos (20 \pi y)
$$

The space steps (with respect to $x$ and $y$ ) are equal to $0.01 \mathrm{~m}$, which means that each furrow is described by 200 cells. We assume that the domain is initially empty, that is $\boldsymbol{u}(0, \boldsymbol{x})=\mathbf{0}$ and $h(0, \boldsymbol{x})=0$.

Let us denote by $h, u$, and $q$ these reference solutions, at the small scale.

\subsubsection{Rainfall test case}

In this case, we impose rainfall on the whole domain with a constant permanent rain intensity $R=8 \times 10^{-4} \mathrm{~m} \mathrm{~s}^{-1}$. The rain discharge is then $Q_{R}=3.2 \times 10^{-3} \mathrm{~m}^{2} \mathrm{~s}^{-1}$. The final time is $T=22.5 \mathrm{~s}$. Note that, since we are interested in the effects of the furrows, we focus on the transitional stage of the flow. Therefore the final time $T$ is chosen such that the outflow discharge is approximately equal to the half of the rain discharge. We assume here that the upstream boundary is a solid wall. We show in Figure 5 the side-view of the water height at the final time. 


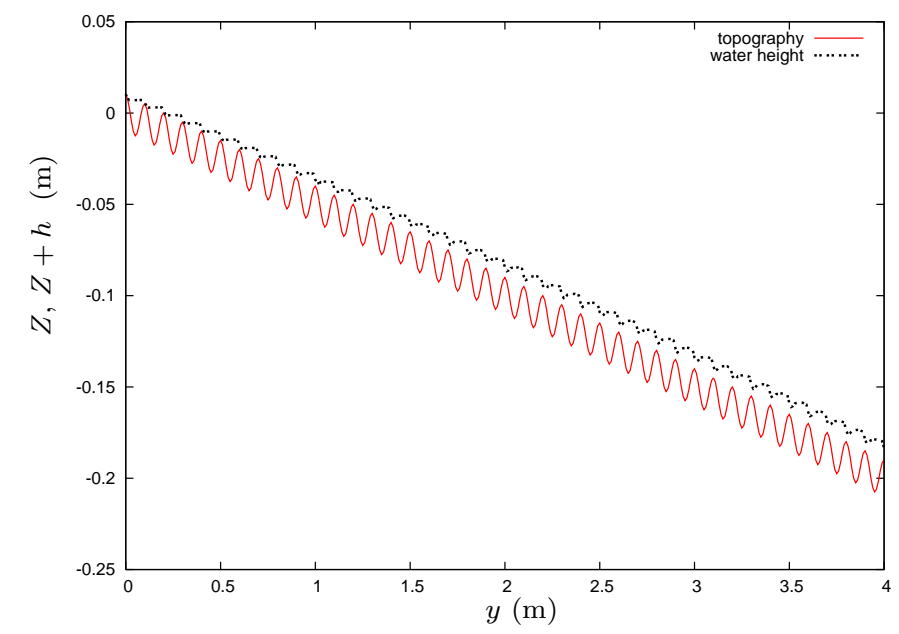

Figure 5: Side-view of the water height at the final time for the rainfall test case

\subsubsection{Inflow test case}

We also consider a permanent inflow from upstream. We prescribe $Q_{I}=3.132 \times 10^{-2} \mathrm{~m}^{2} \mathrm{~s}^{-1}$ as discharge on the inflow boundary. The final time is $T=27.75 \mathrm{~s}$. As for the rainfall test case, the final time was chosen such that the outflow discharge at $T$ is approximately equal to the half of the inflow discharge. We show in Figure 6 the side-view of the water height at the final time.

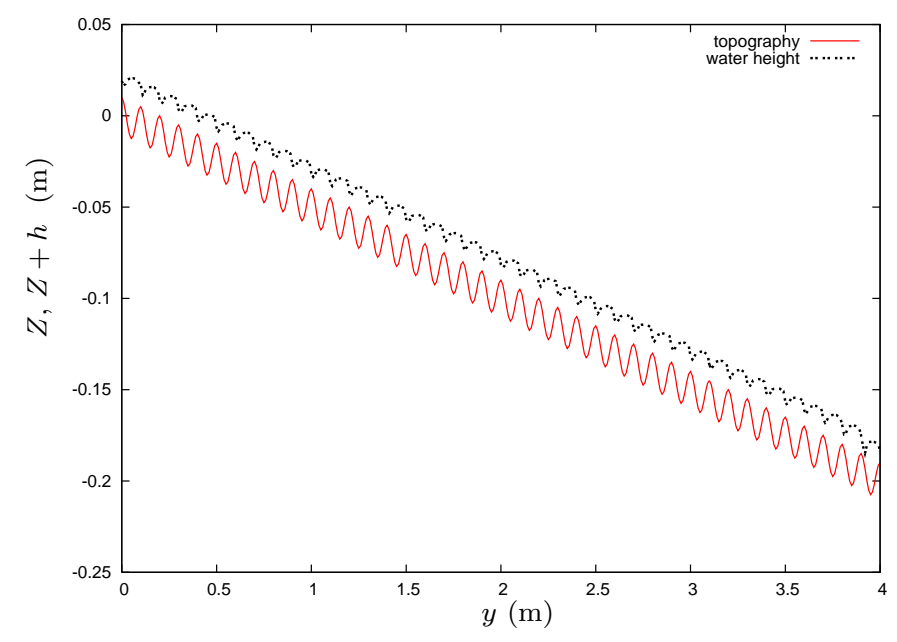

Figure 6: Side-view of the water height at the final time for the inflow test case

\subsection{Numerical comparisons of the models}

In this paragraph, we perform numerical tests on the new model (2.4). The furrows are removed from the topography defined by (4.10). So, the topography is now reduced to an 
inclined plane with the same general slope:

$$
Z(x, y)=-0.05 y
$$

The space step with respect to $y$ is set equal to the wavelength of the furrows, that is 0.1 $\mathrm{m}$. The initial conditions remain unchanged, i.e. $\boldsymbol{u}(0, \boldsymbol{x})=\mathbf{0}$ and $h(0, \boldsymbol{x})=0$. We denote by capital letters $(H, U$ and $Q)$ the solutions of (2.4), at the large scale.

To improve the comparison in the rainfall test, we also compute the solution $(\mathrm{h}, \mathrm{u}, \mathrm{q})$ of the two-layer like system (2.5). The discretization parameters and the topography we use are the same as for system (2.4).

In order to compare the three models $(2.1),(2.4)$ and (2.5), we consider the water height ( $h, H$ and h respectively) and the discharge ( $q, Q$ and q respectively) at the outflow. For this purpose, we first introduce ${\overline{h^{n}}}^{i}$ the average of the reference water height $h$ contained in the furrow $i$ at the time $t^{n}$. We also consider $H_{i}^{n}$ the water height in the furrow $i$ at time $t^{n}$ computed with the model (2.4) for given $K_{0}$ and $C$, and $H_{0 i}^{n}$ in the case $K_{0}=0$ (that is the Shallow Water system on the coarser grid with plane topography and without the new friction term). Next we denote by $e^{H}$ the relative water height error defined by

$$
e^{H}=\left(\frac{\sum_{n=1}^{N} \sum_{i}\left|{\overline{h^{n}}}^{i}-H_{i}^{n}\right|^{2}}{\sum_{n=1}^{N} \sum_{i}\left|\bar{h}^{n}-H_{0 i}^{n}\right|^{2}}\right)^{1 / 2},
$$

where $t^{N}=T$ is the final time of the simulations. This error represents the effect of the new friction term $K(h)$ in system (2.4) on the water height.

Finally we also study the discharge at the outflow: if $q^{n}$ is the value of $q$ (from the resolution of (2.1) with the explicit topography) at time $t^{n}$, for $y=L, Q^{n}$ and $Q_{0}^{n}$ the solutions of (2.4) for given $K_{0}$ and $C$, and $K_{0}=0$ respectively, then the discharge error $e^{Q}$ is defined by:

$$
e^{Q}=\frac{\sum_{n=1}^{N}\left|q^{n}-Q^{n}\right|}{\sum_{n=1}^{N}\left|q^{n}-Q_{0}^{n}\right|} .
$$

The value of $e^{Q}$ shows the influence of the term $K(h)$ on the discharge at the outflow. 


\subsubsection{Rainfall test case}

For the rainfall test case (see paragraph 4.1.1), we present, in Figure 7, the water height error $e^{H}$ between models (2.1) and (2.4) as a function of $K_{0}$ for $C=10$. We started with a step of 0.01 until $K_{0}=0.1$, and then the step is taken equal to 0.1 as the function increases. We remark that the minimum of the error $e^{H} \simeq 0.2518$ is obtained for $K_{0}=0.02$.

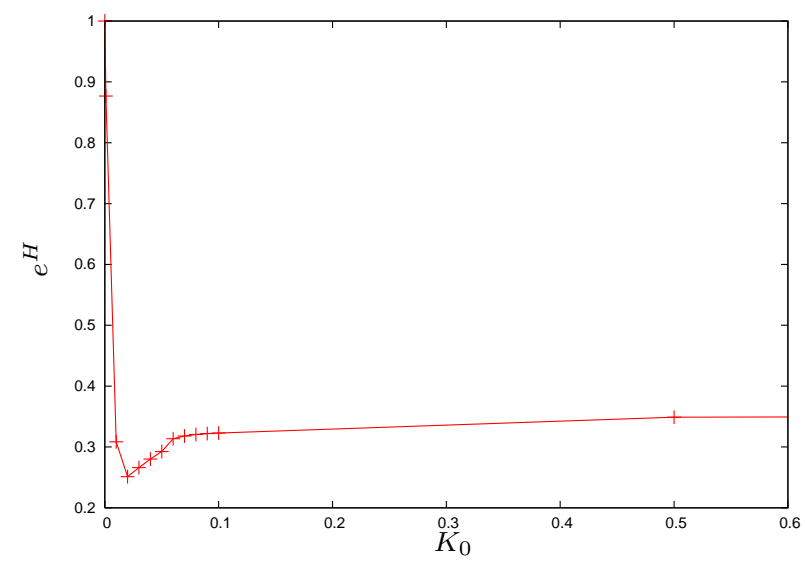

Figure 7: Water height error $e^{H}$ for the model (2.4) for the rainfall test case

Then by optimizing this error with respect to the two parameters $K_{0}$ and $C$, we finally find the minimum $e^{H} \simeq 0.1417$ corresponding to $K_{0}=0.02$ and $C=0.4$. The corresponding discharge error is $e^{Q} \simeq 5.8 \times 10^{-2}$. We notice that the new model (2.4) allows to diminish the $L^{2}$ error on the water height by a factor 7 with respect to the case $K_{0}=0$, showing that the furrow effects are well taken into account.

We now report the results obtained with the two-layer like model (2.5), for the rainfall test. For different Manning's coefficients $k$, Figure 8 shows the water height error $e^{\mathrm{h}}$ (see equality (4.12) replacing the solution $H$ of system (2.4) by the solution h of (2.5)). The objective of this test is to understand how the changes in Manning's coefficient combined with a delay at the beginning of the experiment could represent the effects of the furrows. We note that the minimum is $e^{\mathrm{h}} \simeq 0.0422$ for $k=0.03$. The corresponding discharge error is $e^{Q} \simeq 8.6 \times 10^{-3}$. We notice that the two-layer model allows to diminish the $L^{2}$ error on the water height by a factor 23 with respect to the model (2.4) for the case $K_{0}=0$.

The first conclusion on this rainfall test is that the two proposed models (2.4) and (2.5) are good representations of the effects of the furrows, as they lower the error to the reference solution compared to a plane topography. More precisely, the two-layer like model seems better on this test, but, as we mentioned before, its extension to the general two dimensional case is not obvious. 


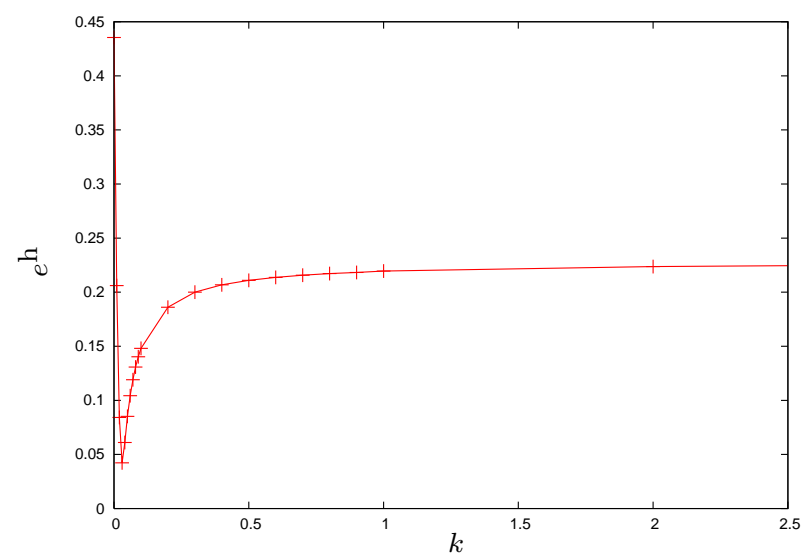

Figure 8: Water height error $e^{\mathrm{h}}$ for the two-layer like model (2.5)

To close this test case, we directly compare the results of the three models. Figure 9 gives the ratio between the outflow discharge obtained by the models and the rain discharge we imposed, as a function of time. We plot four results in the transitory regime: the discharge computed with (2.4) with $K_{0}=0.02$ and $C=0.4$, the discharge with $K_{0}=0$, the solution of (2.5) with $k=0.03$ and the reference solution $q$ (see paragraph 4.1).

Although the domain in the new model (2.4) is an inclined plane, we observe that the additional friction term is able to retain the water for a moment during the rainfall. It is a good approximation to simulate the existence of the furrows.



Figure 9: Ratio between the outflow discharge and the rain discharge for all the models, as a function of time 


\subsubsection{Inflow test case}

Now we consider the inflow test case (see paragraph 4.1.2) and we conduct the same study as for the rainfall test, in order to validate our new model of friction coefficient. Figure 10 shows the water height error $e^{H}$ between models (2.1) and (2.4) as a function of $K_{0}$ for $C=10$. The step on $K_{0}$ is set equal to 0.001 when the error is decreasing, and 0.01 for larger errors. We notice that the minimum of the error is $e^{H} \simeq 0.3211$ for $K_{0}=0.004$. The corresponding discharge error is $e^{Q} \simeq 4.4668 \times 10^{-2}$. Note that our new model (2.4) allows to lower the $L^{2}$ error on the water height by a factor 1.62 with respect to the case $K_{0}$.

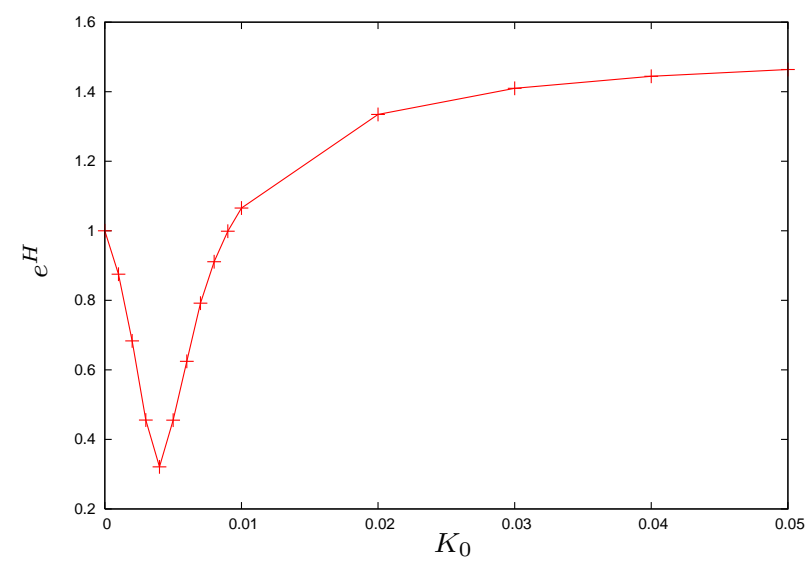

Figure 10: Water height error $e^{H}$ for the model (2.4) for the inflow test case

We also present in Figure 11 the ratio between the outflow discharge $Q^{n}, Q_{0}^{n}$ and the imposed inflow discharge at the transitory regime, for the model (2.4) with $K_{0}=0.004$, $C=10$ and $K_{0}=0$ respectively and for the reference solution $q$ computed in Section 4.1. Here again we observe that the new model simulates well the effect of the furrows.

Remark 4.1. In this part, we did not mention the optimization of the value of $C$ : as the optimized coefficient for $K_{0}$ is ten times smaller than for the rainfall test, the results do not visibly vary when we change $C$.

\subsubsection{Performance of the new model with calibrated coefficients}

In the previous section, the numerical experiments show that our new model (2.4) with the additional friction coefficient to represent the furrows gives good results on the water height and the outflow discharge, compared to the reference solution computed on the explicit topography. But this model needs a calibration of the two coefficients $K_{0}$ and $C$. In this paragraph, we study the robustness of this calibration when we change numerical (the space step) or physical (the slope or the roughness of the domain) parameters. 


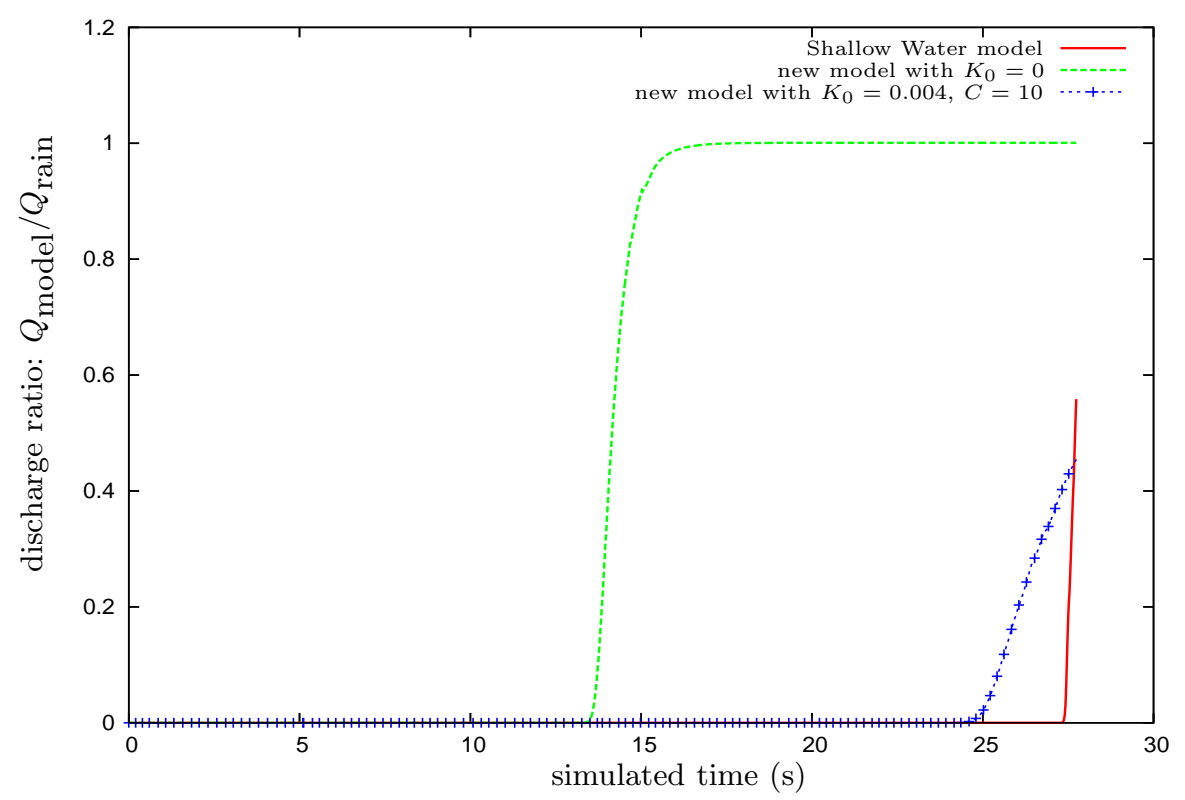

Figure 11: Ratio between the outflow discharge and the imposed inflow discharge for all the models as a function of time

In this part, we only consider the rainfall test case and, as explained before (see paragraph 4.2.1), we choose $K_{0}=0.02$ and $C=0.4$. Consequently, in the following, we study the performance of the model (2.4) with these values.

For a future extension to fully two-dimensional problems, the new model has to predict the flow directions on each grid cell over the area of agricultural fields. As a consequence, the dimension of a grid cell can vary from 1 to $100 \mathrm{~m}^{2}$. So, a grid cell typically contains several furrows. To test for this scale effect, we present in Table 1 the relative errors $e^{H}$ and $e^{Q}$ to the reference solution as functions of the space step. This space step varies from $0.1 \mathrm{~m}$ to $0.4 \mathrm{~m}$ which means that a cell comprises 1 to 4 periods of the furrows. We can notice that the variation of the error $e^{H}$ is small: when the space step is multiplied by 4 , the relative error is multiplied by less than 1.5 .

\begin{tabular}{|c|c|c|c|}
\hline Space step $[\mathrm{m}]$ & $e^{H}$ & $e^{Q}$ & CPU time \\
\hline 0.1 & 0.1417 & $5.8 \times 10^{-2}$ & 0.11 \\
\hline 0.2 & 0.1675 & $7.2865 \times 10^{-2}$ & 0.0574 \\
\hline 0.4 & 0.2024 & $8.963 \times 10^{-2}$ & 0.0255 \\
\hline
\end{tabular}

Table 1: Relative errors on the water height and the flowrate, and CPU times (normalized with respect to the time to compute the reference solution).

At the same time, we present in Table 1 the computational times to compute the solutions of the new model (depending on the space step). The values are normalized with respect 
to the computational time to compute the reference solutions. As we can observe the new model allows to reduce the computational time for at least $90 \%$ and can easily achieve a $97 \%$ decrease under the condition that we tolerate a small error on the solution.

In Table 2, we present the errors $e^{H}$ and $e^{Q}$ for different general slopes of the topography (with a realistic range for agricultural fields), for the calibrated values $K_{0}=0.02$ and $C=0.4$. We note that the new model allows to reduce the $L^{2}$ error on the water height by at least a factor 4 compared to the reference solution, showing that the effects of the furrows are well taken into account.

We complete these results with Table 3 , where, for each slope, we calibrate the coefficients $K_{0}$ and $C$ to get the minimum of the relative error $e^{H}$. Comparing the values of the coefficients and of the errors in Tables 2 and 3, we see that the new model with the calibrated values $K_{0}=0.02, C=0.4$ still yields accurate results.

\begin{tabular}{|c|c|c|c|c|}
\hline slope & $K_{0}$ & $C$ & $e^{H}$ & $e^{Q}$ \\
\hline $2 \%$ & 0.02 & 0.4 & 0.2434 & 0.1855 \\
\hline $5 \%$ & 0.02 & 0.4 & 0.1417 & $5.8 \times 10^{-2}$ \\
\hline $8 \%$ & 0.02 & 0.4 & 0.2194 & 0.187 \\
\hline $11 \%$ & 0.02 & 0.4 & 0.2035 & 0.171 \\
\hline
\end{tabular}

Table 2: Errors $e^{H}$ and $e^{Q}$ for different general slopes of the topography with $K_{0}=0.02$ and $C=0.4$

\begin{tabular}{|c|c|c|c|c|}
\hline slope & $K_{0}$ & $C$ & $e^{H}$ & $e^{Q}$ \\
\hline $2 \%$ & 0.02 & 0.3 & 0.2167 & 0.1134 \\
\hline $5 \%$ & 0.02 & 0.4 & 0.1417 & $5.8 \times 10^{-2}$ \\
\hline $8 \%$ & 0.04 & 0.4 & 0.1205 & $8.1222 \times 10^{-2}$ \\
\hline $11 \%$ & 0.04 & 0.4 & 0.1089 & $6.8554 \times 10^{-2}$ \\
\hline
\end{tabular}

Table 3: Minimum errors $e^{H}$ with the corresponding coefficients $K_{0}, C$ and the values of $e^{Q}$ for different general slopes of the domain

Table 4 shows the errors $e^{H}$ and $e^{Q}$ for different Manning's coefficients (the slope is kept equal to $5 \%$ ), for the calibrated values $K_{0}=0.02$ and $C=0.4$. As for the variation of the slope, in Table 5 , we give the coefficients $K_{0}$ and $C$ that minimize the relative error $e^{H}$ for each Manning's coefficient, and we complete the table with the corresponding error $e^{Q}$. Here again, we see that the results given by our model (2.4) are good compared to the reference solution.

The last results we present are dedicated to water heights and discharges errors at steady state for the Shallow Water model (2.1). The final time of the simulations is then 


\begin{tabular}{|c|c|c|c|c|}
\hline Manning's coefficient $\left[\mathrm{m}^{1 / 3} \mathrm{~s}^{-1}\right]$ & $K_{0}$ & $C$ & $e^{H}$ & $e^{Q}$ \\
\hline 0.001 & 0.02 & 0.4 & 0.0744 & $4.67 \times 10^{-2}$ \\
\hline 0.04 & 0.02 & 0.4 & 0.1417 & $5.8 \times 10^{-2}$ \\
\hline 0.1 & 0.02 & 0.4 & 0.2746 & $9.9691 \times 10^{-2}$ \\
\hline
\end{tabular}

Table 4: Errors $e^{H}$ and $e^{Q}$ for $K_{0}=0.02$ and $C=0.4$ for different Manning's coefficients

\begin{tabular}{|c|c|c|c|c|}
\hline Manning's coefficient $\left[\mathrm{m}^{1 / 3} \mathrm{~s}^{-1}\right]$ & $K_{0}$ & $C$ & $e^{H}$ & $e^{Q}$ \\
\hline 0.001 & 0.02 & 0.4 & 0.0744 & $4.67 \times 10^{-2}$ \\
\hline 0.04 & 0.02 & 0.4 & 0.1417 & $5.8 \times 10^{-2}$ \\
\hline 0.1 & 0.02 & 0.5 & 0.265 & 0.1128 \\
\hline
\end{tabular}

Table 5: Minimum errors $e^{H}$ with the corresponding coefficients $K_{0}, C$ and the values of $e^{Q}$ for different Manning's coefficients

$T=t^{s}=50 \mathrm{~s}$. The two models we consider are, in the one hand, the new model (2.4) with the calibrated coefficients $K_{0}=0.02$ and $C=0.4$, and, on the other hand, the usual Shallow Water model (2.1) with an explicit description of the topography and of the furrows.

We now denote $e_{s}^{H}$ the water height error at steady state defined by

$$
e_{s}^{H}=\left(\frac{\sum_{i}\left|{\overline{h^{s}}}^{i}-H_{i}^{s}\right|^{2}}{\sum_{i}\left|{\overline{h^{s}}}^{i}-H_{0 i}^{s}\right|^{2}}\right)^{1 / 2}
$$

Note that $e_{s}^{H}$ has the same definition as $e^{H}$ (see (4.12)) except that, instead of summing the values of the water heights for all the discrete times, we only consider the time corresponding to the steady state $t^{s}$.

The values we obtain with the new model are $e_{s}^{H} \simeq 0.1765$. Thus we deduce that at the steady state, the new model (2.4) with the calibrated coefficients takes well into account the effects of the furrows and approximates well the Shallow Water model (2.1).

\section{Conclusions}

In this paper, we proposed a new Shallow Water model (2.4) in order to describe the effects of furrows during overland flow without representing them explicitly. The main idea is to include in the classical Shallow Water equations (2.1) the additional friction term (2.3) that takes into account the effects of these furrows. The new model is proposed under the 
assumptions that the flow is fluvial and the direction of the flow is perpendicular to the furrows, parallel to a side of the rectangular domain. We also assumed that there is no infiltration and no soil erosion.

We presented numerical results to show the efficiency and the performance of the new model. We compared it with a two-layer like model, where there is a delay due to the filling of the furrows, and where we are able to assess the behavior of a model with a variable Manning's coefficient. Both models give good results for the rainfall test. We also showed that the calibration of the coefficient of the new model does almost not depend on the slope of the domain (see Table 2 and Table 3 ) and nor on the soil friction coefficient (see Table 4 and Table 5).

The numerical results presented in this paper are encouraging and indicate that the idea could be extended to more complex two-dimensional flows. This is now the main goal of the forthcoming works. Note that, unlike the two-layer model (2.5), the new model (2.4) can easily be generalized to two-dimensional problems. These extensions include the random variations of the height of the furrows. As we already mentioned in the description of (2.3), these variations can be taken into account in the constant $C$ of the additional friction term. The extensions also include the case where the direction of the furrows is not perpendicular to the slope of the domain, in order to study the effects of this direction with respect to the slope.

\section{Acknowledgements}

This work was supported by the ANR project METHODE \#ANR-07-BLAN-0232 (http://www.univ-orleans.fr/mapmo/methode/) and the Région Centre, France. The authors wish to thank the members of the project for fruitful discussions and for many helpful comments. 


\section{References}

[1] E. Audusse. A multilayer Saint-Venant model: derivation and numerical validation. Discrete Contin. Dyn. Syst. Ser. B, 5(2):189-214, 2005.

[2] E. Audusse, F. Bouchut, M.-O. Bristeau, R. Klein, and B. Perthame. A fast and stable well-balanced scheme with hydrostatic reconstruction for shallow water flows. SIAM J. Sci. Comput., 25(6):2050-2065, 2004.

[3] E. Audusse and M.-O. Bristeau. Finite-volume solvers for a multilayer Saint-Venant system. Int. J. Appl. Math. Comput. Sci., 17(3):311-319, 2007.

[4] K. Beven. On undermining the science? Hydrol. Process., 20:2050-2065, 2006.

[5] F. Bouchut. Nonlinear stability of finite volume methods for hyperbolic conservation laws and well-balanced schemes for sources. Frontiers in Mathematics. Birkhäuser Verlag, Basel, 2004.

[6] F. Bouchut. Efficient numerical finite volume schemes for shallow water models. In Ziet lin, V., editor, Nonlinear Dynamics of Rotating Shallow water : Methods and Advances, volume 2 of Edited Series on Advances in Nonlinear Science and Complexity, pages 189 - 256. Elsevier Science, 2007.

[7] M.-O. Bristeau and B. Coussin. Boundary conditions for the shallow water equations solved by kinetics schemes. Inria report, RR-4282, available at http://hal.inria.fr/inria-00072305/en/, 2001.

[8] M. Castro, J. Macías, and C. Parés. A $Q$-scheme for a class of systems of coupled conservation laws with source term. Application to a two-layer 1-D shallow water system. M2AN Math. Model. Numer. Anal., 35(1):107-127, 2001.

[9] F. Darboux, P. Davy, and C. Gascuel-Odoux. Effect of depression storage capacity on overland-flow generation for rough horizontal surfaces: Water transfer distance and scalling. Earth Surf. Process. Landforms, 27(2):177-191, 2002.

[10] A. J.-C. de Saint-Venant. Théorie du mouvement non-permanent des eaux, avec application aux crues des rivières et à l'introduction des marées dans leur lit. C. R. Math. Acad. Sci. Paris, 73:147-154, 1871. [In French].

[11] O. Delestre. Rain water overland flow on agricultural fields simulation. PhD thesis, Université d'Orléans, 2010. [In French], available at http://tel.archivesouvertes.fr/tel-00531377_v1/. 
[12] O. Delestre, S. Cordier, F. Darboux, and F. James. Simulation of rain-water overlandflow. In Eitan Tadmor, Jian-Guo Liu, and Athanasios Tzavaras, editors, Hyp2008 Proceedings of the 12th International Conference on Hyperbolic Problems: Theory, Numerics, Applications, volume 67 of Proceedings of Symposia in Applied Mathematics, pages 537-546, Providence, Rhode Island, USA, jun 9-13 2009. University of Maryland, American Mathematical Society.

[13] Olivier Delestre and François James. Simulation of rainfall events and overland flow. In Proceedings of X International Conference Zaragoza-Pau on Applied Mathematics and Statistics, Jaca, Spain, september 2008, Monografías Matemáticas García de Galdeano, available at http://hal.archives-ouvertes.fr/hal-00426694/fr/, pages 125$135,2009$.

[14] M. Esteves, X. Faucher, S. Galle, and M. Vauclin. Overland flow and infiltration modelling for small plots during unsteady rain: numerical results versus observed values. J. Hydr., 228:265-282, 2000.

[15] S. Ferrari and F. Saleri. A new two-dimensional shallow water model including pressure effects and slow varying bottom topography. M2AN Math. Model. Numer. Anal., 38(2):211-234, 2004.

[16] R. F. Fiedler and J. A. Ramirez. A numerical method for simulating discontinuous shallow flow over an infiltrating surface. Int. J. Num. Meth. Fluids, 32:219-240, 2000.

[17] J.-F. Gerbeau and B. Perthame. Derivation of viscous Saint-Venant system for laminar shallow water; numerical validation. Discrete Contin. Dyn. Syst. Ser. B, 1(1):89$102,2001$.

[18] John E. Gilley, Dennis C. Flanagan, Eugene R. Kottwitz, and Mark A. Weltz. DarcyWeisbach roughness coefficients for overland flow. In Anthony J. Parsons and Athol D. Abrahams, editors, Overland Flow. Hydraulics and Erosion Mechanisms, pages 25-52. University College London Press, London, 1992.

[19] J.M. Greenberg and A.-Y. Le Roux. A well-balanced scheme for the numerical processing of source terms in hyperbolic equation. SIAM J. Num. Ana., 33:1-16, 1996.

[20] V. Jetten, A. de Roo, and D. Favis-Mortlock. Evaluation of field-scale and catchmentscale soil erosion models. Catena, 37:521-541, 1999.

[21] V. Jetten, G. Govers, and R. Hessel. Erosion models: quality of spatial predictions. Hydrol. Process., 17:887-900, 2003. 
[22] C. X. Jin, M. J. M. Römkens, and F. Griffioen. Estimating Manning's roughness coefficient for shallow overland flow in non-submerged vegetative filter strips. Trans. ASAE, 43:1459-1466, 2000.

[23] J. Lhomme. One-dimensional, two-dimensional and macroscopic approaches to urban flood modelling. PhD thesis, Université Montpellier II, 2006. [In French], available at http://tel.archives-ouvertes.fr/tel-00389236/fr/.

[24] F. Marche. Derivation of a new two-dimensional viscous shallow water model with varying topography, bottom friction and capillary effects. Eur. J. Mech. B Fluids, 26(1):49-63, 2007.

[25] O. Planchon, M. Esteves, N. Silvera, and J. Lapetite. Microrelief induced by tillage: measurement and modelling of Surface Storage Capacity. Catena, 46:141-157, 2001.

[26] P. Quinn, K. Beven, P. Chevallier, and O. Planchon. The prediction of hillslope flow paths for distributed hydrological modelling using digital terrain models. Hydrol. Process., 5:59-79, 1991.

[27] Mark W. Smith, Nicholas J. Cox, and Louise J. Bracken. Applying flow resistance equations to overland flows. Progress in Physical Geography, 31(4):363-387, August 2007.

[28] V. Souchère, D. King, J. Daroussin, F. Papy, and A. Capillon. Effects of tillage on runoff directions: consequences on runoff contributing areas within agricultural catchments. J. Hydrol., 206:256-267, 1998.

[29] I. Takken, G. Govers, V. Jetten, L. Nachtergaele, A. Steegen, and J. Poesen. Effect of tillage and runoff and erosion patterns. Soil Tillage Res., 61:55-60, 2001.

[30] D. G. Tarboton. A new method for the determination of flow directions and upslope areas in grid digital elevation models. Water Resour. Res., 33:309-331, 1997. 